\title{
Machine learning assisted analysis of colorimetric assays on paper analytical devices
}

\author{
Bidur Khanal ${ }^{1,2}$, Pravin Pokhrel ${ }^{1}$, Bishesh Khanal $^{2 *}$, Basant Giri ${ }^{*}$ \\ ${ }^{1}$ Center for Analytical Sciences, Kathmandu Institute of Applied Sciences, Kathmandu, Nepal \\ ${ }^{2}$ NepAl Applied Mathematics and Informatics Institute for Research, Kathmandu, Nepal
}

*For correspondence

Email: bgiri@kias.org.np (Dr. Basant Giri)

Email: bishesh.khanal@naamii.org.np (Dr. Bishesh Khanal) 


\section{Supplementary information}

Table S1: Average Accuracy across Food color and Pesticide Test Sets in Various settings

Test Accuracy Results on Separate Test Sets

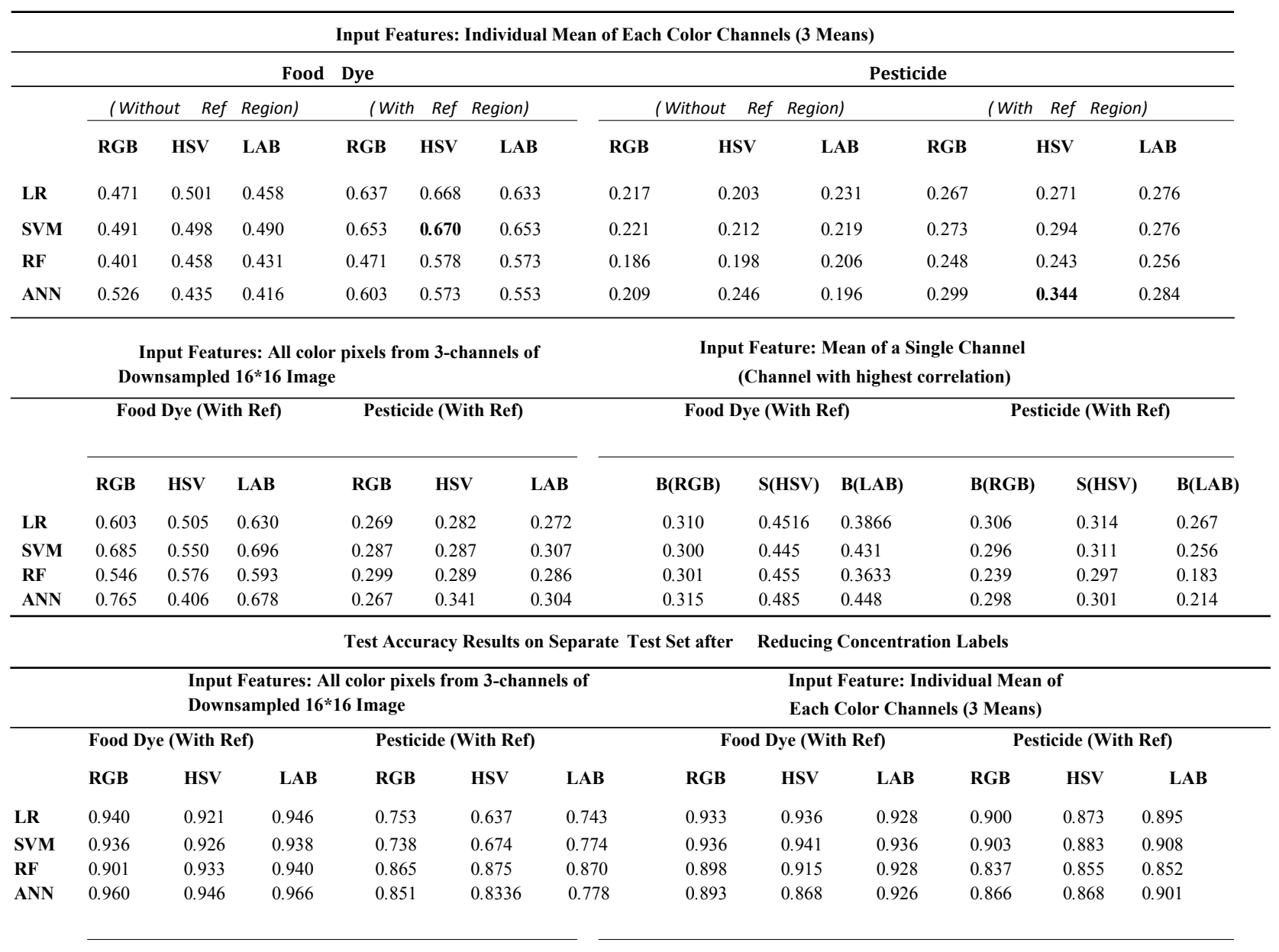


Table S2: Cross Validation Accuracy (in mean and standard deviation) across Food color and Pesticide in Various settings

Cross-Validation Accuracy Results (mean $\pm s t d$ )

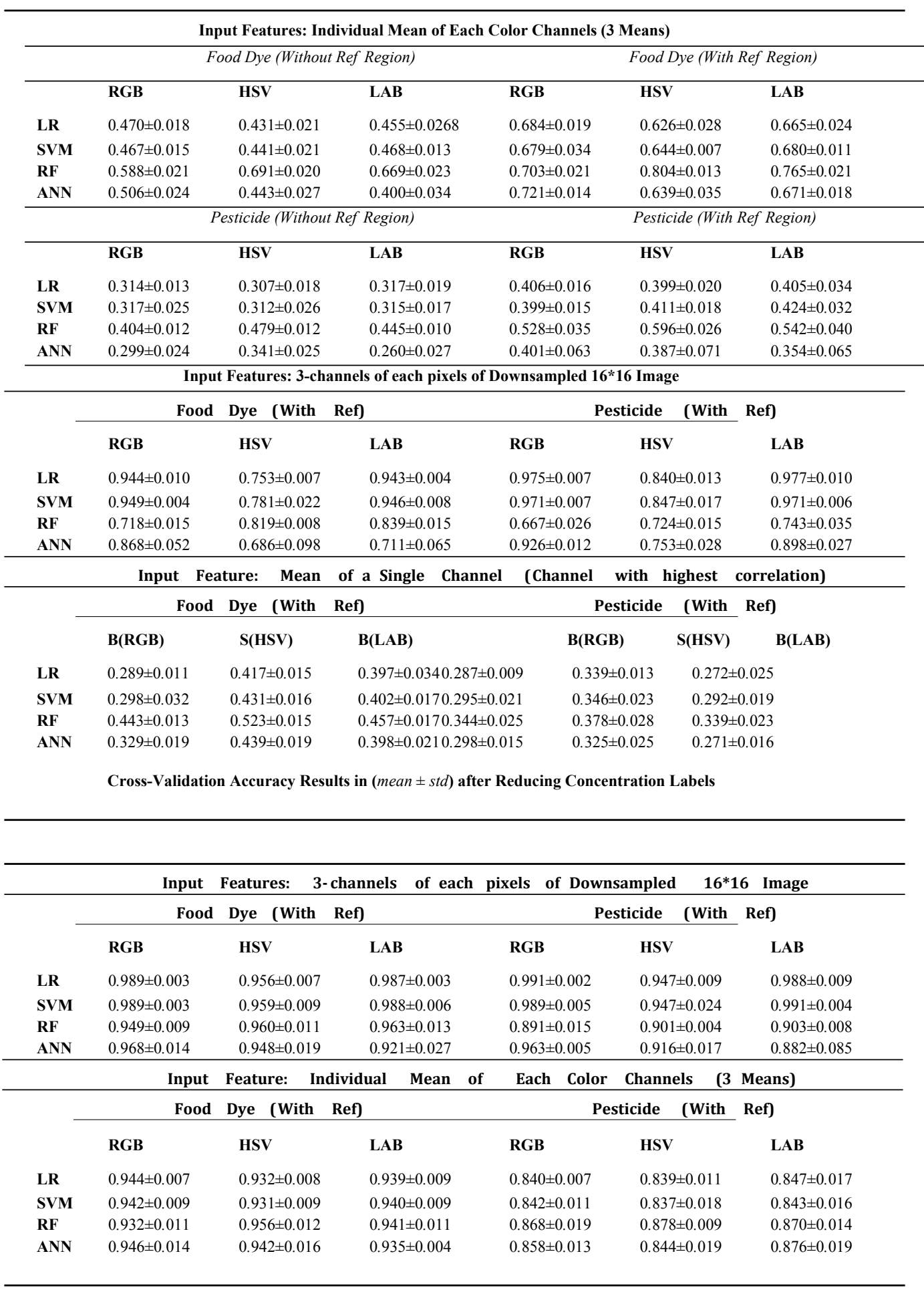

